\title{
Rescuing the nonjet (NJ) azimuth quadrupole from the flow narrative
}

\author{
Thomas A. Trainor ${ }^{1}$ \\ ${ }^{1}$ CENPA 354290 University of Washington, Seattle, Washington, USA
}

\begin{abstract}
According to the flow narrative commonly applied to high-energy nuclear collisions a cylindrical-quadrupole component of 1D azimuth angular correlations is conventionally denoted by quantity $v_{2}$ and interpreted to represent elliptic flow. Jet angular correlations may also contribute to $v_{2}$ data as "nonflow" depending on the method used to calculate $v_{2}$, but 2D graphical methods are available to insure accurate separation. The nonjet (NJ) quadrupole has various properties inconsistent with a flow interpretation, including the observation that NJ quadrupole centrality variation in A-A collisions has no relation to strongly-varying jet modification ("jet quenching") in those collisions commonly attributed to jet interaction with a flowing dense medium. In this presentation I describe isolation of quadrupole spectra from pt-differential $v_{2}\left(p_{t}\right)$ data from the RHIC and LHC. I demonstrate that quadrupole spectra have characteristics very different from the single-particle spectra for most hadrons, that quadrupole spectra indicate a common boosted hadron source for a small minority of hadrons that "carry" the NJ quadrupole structure, that the narrow source-boost distribution is characteristic of an expanding thin cylindrical shell (strongly contradicting hydro descriptions), and that in the boost frame a single universal quadrupole spectrum (Lévy distribution) on transverse mass $m_{t}$ accurately describes data for several hadron species scaled according to their statistical-model abundances. The quadrupole spectrum shape changes very little from RHIC to LHC energies. Taken in combination those characteristics strongly suggest a unique nonflow (and nonjet) QCD mechanism for the NJ quadrupole conventionally represented by $v_{2}$.
\end{abstract}

\section{Introduction}

The flow narrative, believed by some to be of central importance to high-energy nuclear collisions $[1,2]$, is based primarily on $v_{2}$ data interpreted to represent elliptic flow - azimuthal modulation of radial flow of a locally-thermalized bulk medium in non-central $A$ - $A$ collisions. Earlier versions referred to more-central $A-A$ collisions at higher collision energies. More recently, "collectivity" in small systems $(p-p, p-A)$ has been claimed as well, based on certain LHC data [3, 4]. However, evidence strongly contradicting the flow narrative has accumulated over the past ten years: Differential analysis of $p_{t}$ spectra from $200 \mathrm{GeV} \mathrm{Au}-\mathrm{Au}$ collisions reveals no evidence for radial flow - the blast-wave model said to measure radial flow responds 
instead to a predicted and observed strong jet contribution to spectra [5]. Measurements of $p_{t}$-integral nonjet $(\mathrm{NJ}) v_{2}$, based on model fits to $2 \mathrm{D}$ angular correlations that exclude a jet contribution ("nonflow") [6], reveal $v_{2}$ systematics uncorrelated with "jet quenching" [7] contradicting claims for a dense bulk medium [8,9]. Equivalent systematic $v_{2}$ trends are observed for high-multiplicity $A$ - $A$ collisions and for $p-p$ collisions down to negligible particle densities [10]. There is no evidence for a QCD phase transition or changing equation of state.

In this talk I present quadrupole spectra inferred from $v_{2}\left(p_{t}, b\right)$ data that further contradict the flow narrative. Quadrupole spectra from recent $\mathrm{LHC} \mathrm{Pb}-\mathrm{Pb}$ data are compared to those from previous analysis of $200 \mathrm{GeV} \mathrm{Au-Au} \mathrm{data} \mathrm{[11].} \mathrm{Quadrupole} \mathrm{spectra} \mathrm{reveal} \mathrm{a} \mathrm{hadron}$ source boost incompatible with Hubble expansion of a flowing bulk medium, and the spectrum shape is very different from the single-particle (SP) spectrum for most final-state hadrons.

\section{Quadrupole Spectrum Definition}

$p_{t}$-differential $v_{2}$ is defined as the ratio of the quadrupole $(m=2)$ Fourier amplitude of the event-wise azimuth-dependent SP spectrum to the azimuth-averaged SP spectrum.

$$
v_{2}\left(p_{t}, b\right)=\frac{V_{2}\left(p_{t}, b\right)}{\bar{\rho}_{0}\left(p_{t}, b\right)}=\frac{V_{2}\{2 D\}\left(p_{t}\right)+\text { jet contribution }}{\left(N_{\text {part }} / 2\right) S_{N N}\left(p_{t}\right)+N_{b i n} r_{A A}\left(p_{t}, b\right) H_{N N}\left(p_{t}\right)} .
$$

As defined that ratio may include two jet contributions: (a) jet-related angular correlations in the numerator and (b) SP spectrum hard component $H_{A A}\left(p_{t}, b\right) \equiv r_{A A}\left(p_{t}, b\right) H_{N N}\left(p_{t}\right)$ in the denominator. The $v_{2}$ definition assumes that almost all hadrons "carry" the quadrupole correlation component and therefore are described by the same SP spectrum that should then cancel in the $v_{2}$ ratio. The NJ quadrupole Fourier amplitude, assuming source-boost azimuth distribution $\Delta y_{t}\left(\phi_{r}, b\right)=\Delta y_{t 0}(b)+\Delta y_{t 2}(b) \cos \left(2 \phi_{r}\right)$, can be expressed as [11]

$$
V_{2}\left(y_{t}, b\right)=\frac{1}{2 \pi} \int_{-\pi}^{\pi} d \phi \bar{\rho}_{0}\left(y_{t}, b, \phi_{r}\right) \cos \left(2 \phi_{r}\right) \approx p_{t}^{\prime} \frac{\Delta y_{t 2}(b)}{2 T_{2}} \bar{\rho}_{2}\left[y_{t}, b ; \Delta y_{t 0}(b)\right],
$$

where $\phi_{r}$ is $\phi$ relative to a reference angle, $p_{t}^{\prime}$ is $p_{t}$ in the boost frame, transverse rapidity is $y_{t} \equiv \ln \left[\left(p_{t}+m_{t}\right) / m_{h}\right]$ and $\bar{\rho}_{2}\left[y_{t}, b ; \Delta y_{t 0}(b)\right]$ is the spectrum for those hadrons carrying the quadrupole correlation component, which may or may not be equivalent to $\bar{\rho}_{0}\left(y_{t}, b\right)$.

\section{Ideal Hydro}

The following simple system illustrates some implications of Eq. (2). The source-boost distribution, which should be broad for Hubble expansion of a bulk medium, is assumed to be a single fixed value $\Delta y_{t 0}$ for each collision system. The quadrupole spectrum $\bar{\rho}_{2}\left[y_{t}, b ; \Delta y_{t 0}(b)\right]$ is assumed to coincide with SP spectrum $\bar{\rho}_{0}\left[p_{t}, b ; \Delta y_{t 0}(b)\right]$ including centrality-dependent radial flow [12] measured by $\Delta y_{t 0}(b)$. In that case $v_{2}\left(p_{t}\right) \approx p_{t}^{\prime}\left(\Delta y_{t 2} / 2 T_{2}\right)$ and $v_{2}\left(p_{t}\right) / p_{t} \propto p_{t}^{\prime} / p_{t}$. Figure 1 (a) shows $p_{t}^{\prime}$ in the boost frame vs lab $p_{t}$ for three hadron species. Figure 1 (b) shows ratio $p_{t}^{\prime} / p_{t}$ vs $y_{t}$ (with proper hadron mass) as a universal curve common to all hadron species, with shape determined solely by fixed source boost $\Delta y_{t 0}=0.6$. Figure 1 (c) shows panel (a) adjusted to anticipate $200 \mathrm{GeV} v_{2}\left(p_{t}\right)$ data, and panel (d) shows the equivalent for ratio $v_{2}\left(p_{t}\right) / p_{t}$. In each case viscous-hydro predictions for $200 \mathrm{GeV} \mathrm{Au-Au} \mathrm{collisions} \mathrm{(dotted}$ curves) [13] shown for comparison exhibit striking differences from the "ideal-hydro" example. This exercise illustrates "mass ordering" at lower $p_{t}$ in the conventional plotting format of panel (a). The quantitative source-boost distribution that hydro theory actually predicts (broad or narrow?) is more easily tested in the format of panel (d) than in (a) or (c). 

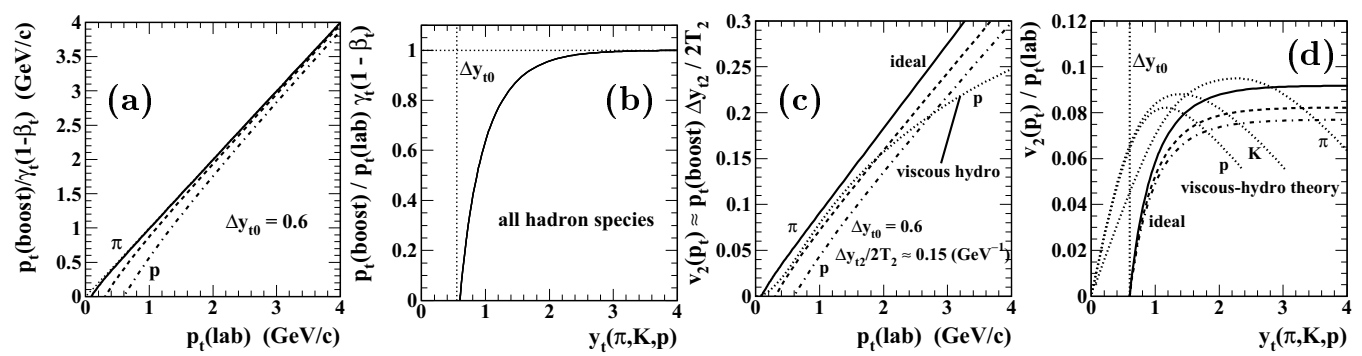

Figure 1. "Ideal-hydro" kinematic trends assuming single value of source boost $\Delta y_{t 0}$ and quadrupole spectrum equal to SP spectrum [11]. Viscous-hydro theory trends [13] are included for comparison.

\section{$4200 \mathrm{GeV}$ Au-Au Quadrupole Spectra}

Quadrupole spectra can be inferred from $v_{2}\left(p_{t}, b\right)$ data in a few steps. Figure 2 (a) shows $0-80 \%$ central $v_{2}\left(p_{t}\right)$ data for three hadron species in the conventional plotting format [14, 15]. "Mass ordering" below $1.5 \mathrm{GeV} / \mathrm{c}$ is said to indicate a hydro mechanism. Curves representing "ideal hydro" cross the top edge. A viscous-hydro theory curve for protons is indicated by $\mathrm{R}$ [13]. Curves passing through data at higher $p_{t}$ are explained below. Figure 2 (b) shows the same data in the form $v_{2}\left(p_{t}\right) / p_{t}$ (lab) vs $y_{t}$ with proper mass for each hadron species. The ideal-hydro curves go to a constant value for higher $y_{t}$, and the data trends for three hadron species share a common zero intercept that can be identified with fixed source boost $\Delta y_{t 0} \approx 0.6$. Figure 2 (c) confirms that the (dotted) viscous-hydro theory curve is strongly falsified by Lambda (or proton) data. Figure 2 (d) shows the quadrupole Fourier coefficient as the product $V_{2}\left(y_{t}\right)=\bar{\rho}_{0}\left(y_{t}\right) v_{2}\left(y_{t}\right)$ obtained via SP spectra for three hadron species [11].
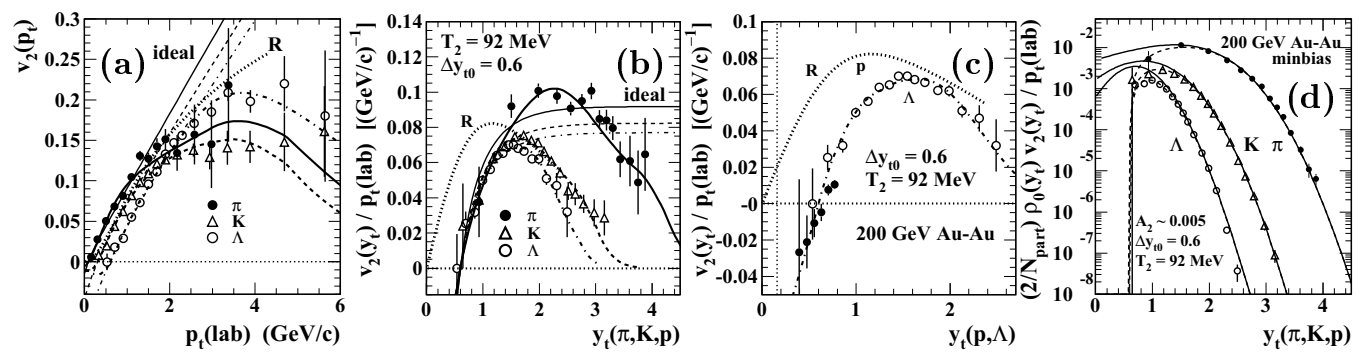

Figure 2. $v_{2}\left(p_{t}\right)$ data for three hadron species from $0-80 \% 200 \mathrm{GeV} \mathrm{Au}-\mathrm{Au}$ collisions $[14,15]$ processed to obtain $V_{2}\left(y_{t}\right)=\bar{\rho}_{0}\left(y_{t}\right) v_{2}\left(y_{t}\right) \propto p_{t}^{\prime}\left(y_{t}\right) \bar{\rho}_{2}\left(y_{t} ; \Delta y_{t 0}\right)$ quadrupole spectra as in Ref. [11].

To obtain final quadrupole spectra from the data in Fig. 2 (d) three more steps are required. The spectra are multiplied by $p_{t}$ (lab) $/ p_{t}$ (boost) determined exactly by inferred $\Delta y_{t 0}=0.6$, shifted left by $\Delta y_{t 0}$ from lab frame $y_{t}$ to boost frame $y_{t}^{\prime}$ and finally transformed to boost-frame $m_{t}^{\prime}$ with the appropriate Jacobian. The result is shown in Figure 3 . The spectra, rescaled by their statistical-model abundances relative to pions [16], lie on a common locus (solid curve) with slope parameter $T_{2}=92 \mathrm{MeV}$ and Lévy exponent $n_{2}=14$ dramatically different from the hadron SP spectrum with $T_{0}=145 \mathrm{MeV}$ and $n_{0}=12$ [5]. The solid curve, back-transformed by reversing the sequence, gives the curves passing through data in Fig. 2. 


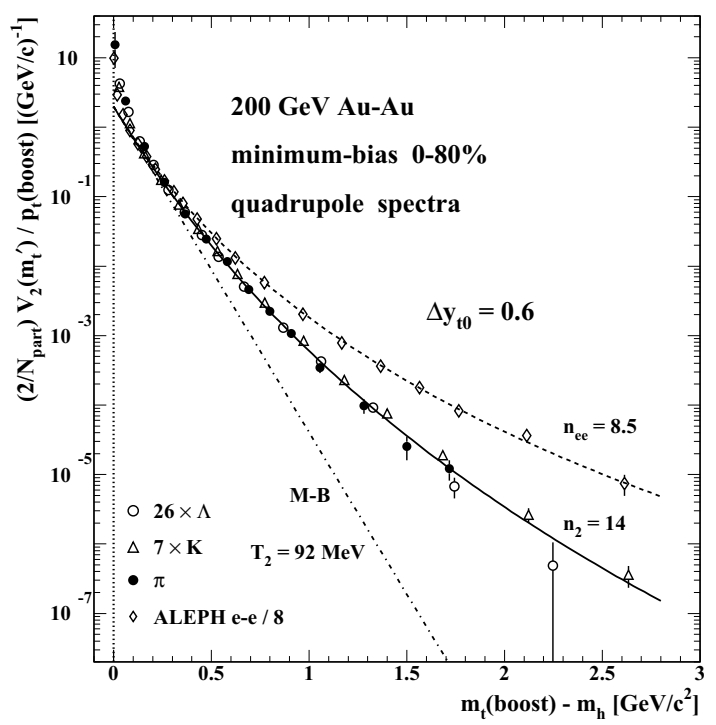

Figure 3. Quadrupole spectra from $200 \mathrm{GeV}$ $\mathrm{Au}-\mathrm{Au}$ collisions for three hadron species (points in legend) rescaled by statistical-model abundances relative to pions (factors noted in the legend) plotted on $m_{t}^{\prime}$ in the boost frame. The solid curve is a Lévy distribution with $T_{2}=92 \mathrm{MeV}$ (dash-dotted curve) and exponent $n_{2}=14$ very different from $\mathrm{Au}-\mathrm{Au}$ SP spectrum values $T_{0}=145 \mathrm{MeV}$ and $n_{0}=12[5]$. The dashed curve and data are derived from the fragment $p_{t}$ spectrum (perpendicular to the dijet axis) for $91 \mathrm{GeV}$ LEP dijets [17] also exhibiting $T \approx 90 \mathrm{MeV}$.

\section{2.76 TeV Pb-Pb Quadrupole Spectra}

Figure 4 (a,b) shows $v_{2}\left(p_{t}, b\right)$ data vs $p_{t}$ for pions and protons from seven centralities of 2.76 $\mathrm{TeV} \mathrm{Pb}-\mathrm{Pb}$ collisions [18]. $200 \mathrm{GeV} v_{2}\left(p_{t}, b\right)$ data are presented in Ref. [19] for comparison. Figure 4 (c) shows proton data plotted as in Fig. 2 (b) but rescaled by $p_{t}$-integral values $v_{2}(b)$ [20]. The dashed curve is the $200 \mathrm{GeV}$ equivalent. The $2.76 \mathrm{TeV}$ data reveal significant variation of source boost $\Delta y_{t 0}(b)$ with centrality. Figure $4(\mathrm{~d})$ shows the same data with all centralities boosted (shifted on $y_{t}$ ) to coincide with the $30-40 \%$ data. Within uncertainties all centralities follow the same locus that coincides also with the $200 \mathrm{GeV}$ dashed curve.
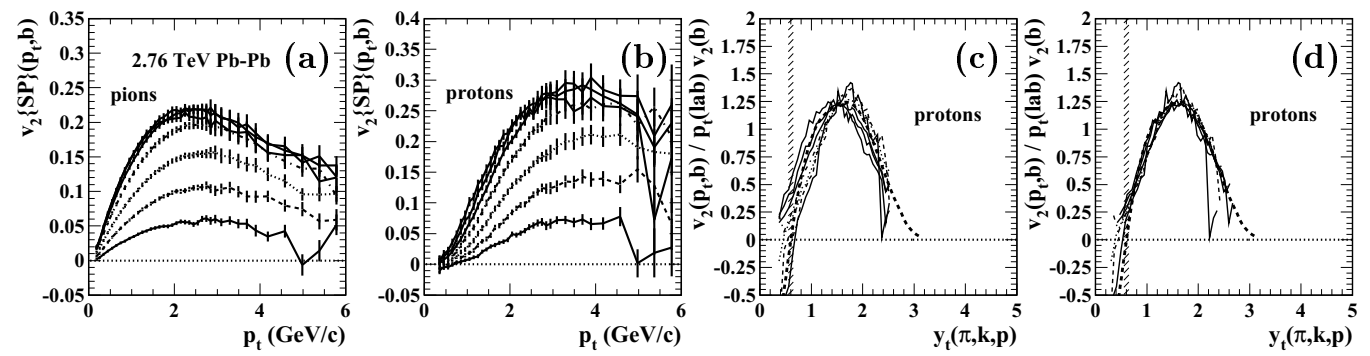

Figure 4. $v_{2}\left(p_{t}, b\right)$ data vs $p_{t}$ for pions and protons from $2.76 \mathrm{TeV} \mathrm{Pb}-\mathrm{Pb}$ collisions [18].

Figure $5(\mathrm{a}, \mathrm{b})$ shows SP spectra for pions and protons from p-p collisions (open points) and from $30-40 \%$ central $2.76 \mathrm{TeV} \mathrm{Pb}-\mathrm{Pb}$ (solid dots) as the average of $0-5 \%$ and $60-80 \%$ central collisions with two issues [21]: (a) The $\mathrm{Pb}-\mathrm{Pb}$ pion spectrum normalized by $N_{\text {part }} / 2$ must be divided by factor 1.65 to coincide with the $p$ - $p$ spectrum at lower $p_{t}$ in accord with $200 \mathrm{GeV}$ data [5]. (b) The $\mathrm{Pb}-\mathrm{Pb}$ proton spectrum normalized by $N_{\text {part }} / 2$ (solid dots) is divided by the same factor but falls substantially below (factor 2 ) the $p$ - $p$ spectrum at lower $p_{t}$ suggesting significant uncorrected inefficiency in that interval. Figure 5 (c) shows the equivalent of Fig. 2 
(d) for four hadron species. The dashed curves through those data are the dashed curves for $200 \mathrm{GeV} v_{2}\left(p_{t}, b\right) / p_{t}(\mathrm{lab})$ multiplied by the $\mathrm{Pb}-\mathrm{Pb} \mathrm{SP}$ spectra rescaled by $1 / 1.65$. Figure $5(\mathrm{~d})$ shows those data multiplied by factor $p_{t}(\mathrm{lab}) / p_{t}$ (boost) and transformed to the boost frame (shifted left by $\Delta y_{t 0}=0.6$ ).
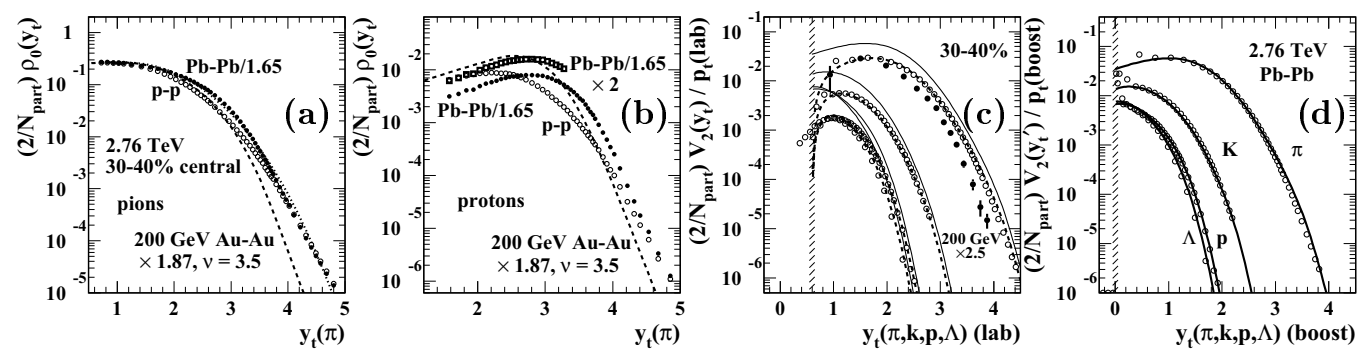

Figure 5. SP spectra for pions (a) and protons (b) from $30-40 \%$ central $2.76 \mathrm{TeV} \mathrm{Pb}-\mathrm{Pb}$ collisions (solid points). Quadrupole spectra for the same system in the lab frame (c) and boost frame (d).

Figure 6 shows data and curves in Fig. 5 (d) transformed to $m_{t}^{\prime}$ in the boost frame via the proper Jacobian and rescaled by statistical-model abundances relative to pions (points in legend). The pion data fall on the bold solid curve with $T_{2}=94 \mathrm{MeV}$ and $n_{2}=12$. The $200 \mathrm{GeV}$ quadrupole spectrum data (inverted solid triangles and thin solid curve) are shown multiplied by factor 2.5 expected from observed energy scaling of $v_{2}$ and $\bar{\rho}_{0}$. Slope parameters $T_{2}$ are not significantly different, but the Lévy exponent decreases significantly at the higher energy consistent with the trend for SP spectrum soft component $\hat{S}_{0}\left(m_{t}\right)$ [21, 22]. Just as at RHIC energies there is a great difference between quadrupole spectra and SP spectra. SP spectrum soft component $\hat{S}_{0}\left(m_{t}^{\prime}\right)$ for $2.76 \mathrm{TeV} p-p$ collisions plotted on $m_{t}^{\prime}$ is shown as the dashed curve. The spectrum for protons (and Lambdas) falls substantially below the bold solid curve for $m_{t}^{\prime}<0.7 \mathrm{GeV} / c^{2}$, consistent with the spectrum result in Fig. 5 (b). The dotted curves for kaons and protons are the $200 \mathrm{GeV}$ dashed curves in Fig. 5 (c) derived from the solid curve in Fig. 3 processed in this case with the corresponding $2.76 \mathrm{TeV}$ SP spectra.

\section{Summary}

Quadrupole spectra for identified hadrons, which may involve only a small fraction of finalstate particles that actually "carry" the quadrupole component, can be extracted from $p_{t^{-}}$ differential $v_{2}\left(p_{t}, b\right)$ data by a simple sequence of transformations given the availability of matching single-particle hadron spectra. The sequence leads to determination of a hadron source-boost distribution consistent with a single value $\Delta y_{t 0}$ (for a given collision system) that is inconsistent with the broad distribution expected for Hubble expansion of a flowing bulk medium. Quadrupole spectra at $2.76 \mathrm{TeV}$ are remarkably similar to those for $200 \mathrm{GeV}$ and very different from single-particle hadron spectra, contradicting a basic assumption of the flow narrative that almost all hadrons must participate in such flows. These new results, combined with related trends observed over the past ten years, strongly suggest that the nonjet azimuth quadrupole does not represent a hydrodynamic flow. The NJ quadrupole may instead be the manifestation of a QCD mechanism similar to QED antenna radiation. 


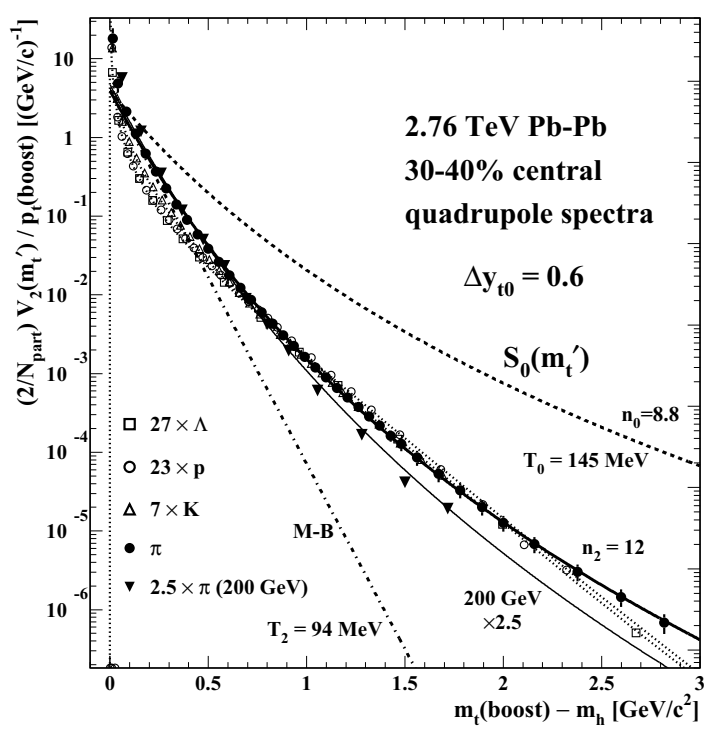

Figure 6. Quadrupole spectra from $2.76 \mathrm{TeV}$ $\mathrm{Pb}-\mathrm{Pb}$ collisions for four hadron species (points in legend) rescaled by statistical-model abundances relative to pions (factors noted in the legend) plotted on $m_{t}^{\prime}$ in the boost frame. The solid curve is a Lévy distribution with $T_{2}=94 \mathrm{MeV}$ (dash-dotted curve) and exponent $n_{2}=12$, very different from $p-p$ SP spectrum soft component $\hat{S}_{0}\left(m_{t}^{\prime}\right)$ (dashed curve) values $T_{0}=145 \mathrm{MeV}$ and $n_{0}=8.8[5]$. The $200 \mathrm{GeV}$ quadrupole spectrum multiplied by anticipated energy-scaling factor 2.5 is plotted as the thin solid curve and inverted solid triangles

\section{References}

[1] D. Teaney, J. Lauret and E. V. Shuryak, Phys. Rev. Lett. 86, 4783 (2001).

[2] M. Gyulassy and L. McLerran, Nucl. Phys. A 750, 30 (2005).

[3] CMS Collaboration, JHEP 1009, 091 (2010), arXiv:1009.4122.

[4] T. A. Trainor and D. T. Kettler, Phys. Rev. C 84, 024910 (2011).

[5] T. A. Trainor, Int. J. Mod. Phys. E 17, 1499 (2008).

[6] D. T. Kettler (STAR collaboration), Eur. Phys. J. C 62, 175 (2009).

[7] J. Adams et al. (STAR Collaboration), Phys. Rev. Lett. 91, 172302 (2003).

[8] T. A. Trainor, J. Phys. G 37, 085004 (2010).

[9] T. A. Trainor, D. T. Kettler, D. J. Prindle and R. L. Ray, J. Phys. G 42, 025102 (2015).

[10] T. A. Trainor and D. J. Prindle, Phys. Rev. D 93, no. 1, 014031 (2016).

[11] T. A. Trainor, Phys. Rev. C 78, 064908 (2008).

[12] B. I. Abelev et al. (STAR Collaboration), Phys. Rev. C 79, 034909 (2009).

[13] P. Romatschke and U. Romatschke, Phys. Rev. Lett. 99, 172301 (2007).

[14] C. Adler et al. (STAR Collaboration), Phys. Rev. C 66, 034904 (2002).

[15] J. Adams et al. (STAR Collaboration), Phys. Rev. Lett. 92, 052302 (2004).

[16] F. Becattini, M. Gazdzicki and J. Sollfrank, Eur. Phys. J. C 5, 143 (1998).

[17] D. Buskulic et al. [ALEPH Collaboration], Z. Phys. C 55, 209 (1992).

[18] B. B. Abelev et al. (ALICE Collaboration), JHEP 1506, 190 (2015).

[19] D. T. Kettler, D. J. Prindle and T. A. Trainor, Phys. Rev. C 91, 064910 (2015).

[20] K. Aamodt et al. (ALICE Collaboration), Phys. Rev. Lett. 105, 252302 (2010)

[21] T. A. Trainor, arXiv:1603.01337 [hep-ph].

[22] B. B. Abelev et al. (ALICE Collaboration), Phys. Lett. B 736, 196 (2014). 